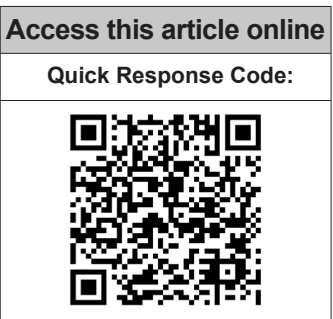

Website:

www.jponline.org

DOI:

10.4103/JLP.JLP_167_16
Anandaloke Sonoscan Centre, ${ }^{2}$ Department of Pathology, North Bengal Medical College, Siliguri, 'Department of Pathology, College of Medicine and Sagore Dutta Hospital, Kolkata, West Bengal, India

Address for correspondence: Dr. Subrata Pal, Gobindanagar, Kenduadihi, Bankura, West Bengal, India. E-mail: subratapal1985@ gmail.com

Submission: 19-12-2016 Accepted: 13-11-2017

\title{
Well differentiated papillary mesothelioma of abdomen- a rare case with diagnostic dilemma
}

\author{
Aniruddha Saha, Palash Kumar Mandal' ${ }^{1}$, Anupam Manna, Kalyan Khan², Subrata Pal ${ }^{1}$
}

\section{Abstract:}

Well-differentiated papillary mesothelioma is a rare tumor occurring predominantly in the peritoneum of young women, a few with history of asbestos exposure. A 28-year-old woman presented with ascites and pain abdomen. Ultrasonography and computed tomography scan of the abdomen revealed a mass in the retroperitoneum measuring $15 \mathrm{~cm} \times 12 \mathrm{~cm}$. Histopathological examination along with immunohistochemistry (IHC) confirmed it to be a papillary mesothelioma in the peritoneum. It is difficult to differentiate from more common malignant mesothelioma and papillary adenocarcinoma, which also have poorer prognosis. The difficulty can be resolved by clinico-radiological correlation along with histopathological examination and IHC.

Key words:

Immunohistochemistry, peritoneum, well-differentiated papillary mesothelioma

\section{Introduction}

W ell-differentiated papillary mesotheliomas (WDPMs) of the peritoneum are uncommon; approximately 50 cases have been reported till date. ${ }^{[1-5]}$ Approximately $75 \%$ of the tumors occurred in females who are usually of reproductive age but occasionally postmenopausal. [2] WDPM is usually an incidental finding at laparotomy, but rare tumors have been associated with abdominal pain, ascites, menorrhagia, etc., The presence of ascites and other symptoms, however, is much more common in malignant mesothelioma. ${ }^{[3]}$ Some of the patients had a history of asbestos exposure. ${ }^{[4]}$ We report a case of WDPM in a young woman without asbestos exposure.

\section{Case Report}

A 27-year-old woman presented with pain abdomen and distension. There was no history of exposure to asbestos or occupation in the construction industry.

This is an open access article distributed under the terms of the Creative Commons Attribution-NonCommercial-ShareAlike 3.0 License, which allows others to remix, tweak, and build upon the work non-commercially, as long as the author is credited and the new creations are licensed under the identical terms.

For reprints contact: reprints@medknow.com
The ultrasonography of whole abdomen showed an abdomino-pelvic mass along with ascites. Ascitic fluid examination was done and showed few cellular fragments of mesothelial cells admixed with lymphocytes. Abdominal computed tomography (CT) scan showed mass measuring $14 \mathrm{~cm} \times 11 \mathrm{~cm}$, but the site of origin could not be identified and possibility of the left adnexal mass was suggested [Figure 1a].

An exploratory laparotomy was carried out and the mass removed. During the procedure, a granular papillary lesion was incidentally found at the peritoneum adherent to the uterus mimicking adnexal mass. On gross, an irregular gray-white mass measuring $15 \mathrm{~cm} \times 12 \mathrm{~cm}$ and showing a granular papillary projection on the surface was noted [Figure 1b]. Histopathological examination revealed a neoplasm composed of broad papillae with edematous fibrous cores and lined by uniform cuboidal cells showing moderate pale to eosinophilic cytoplasm and central nuclei with fine chromatin. Areas of vascular invasion and psammoma bodies

How to cite this article: Saha A, Mandal PK, Manna A, Khan K, Pal S. Well differentiated papillary mesothelioma of abdomen- a rare case with diagnostic dilemma. J Lab Physicians 2018;10:248-50. 


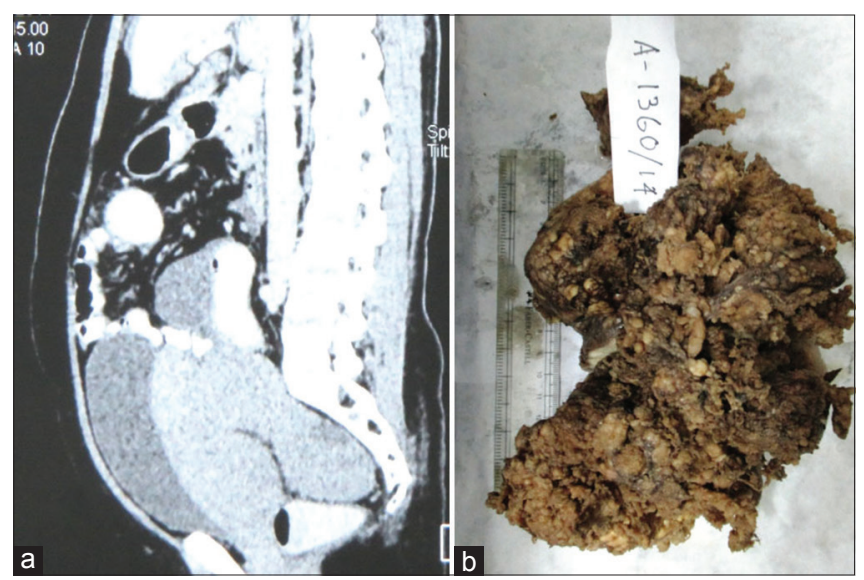

Figure 1: Computed tomography scan showing a intra-abdominal mass measuring $14 \mathrm{~cm} \times 11 \mathrm{~cm}$, without any definite site of origin. (b): Gross image of specimen of papillary mesothelioma

were also noted. There was no evidence of atypia, mitosis, or necrosis [Figure $2 \mathrm{a}, \mathrm{b}$ and $\mathrm{c}$ ].

Immunohistochemistry (IHC) revealed a positive staining pattern for vimentin, calretinin [Figure 2d], Wilms tumor protein 1 (WT 1), and cytokeratin (focal). The Ki-67 proliferation index was about $1 \%$. Desmin, Ber-EP4, and chromogranin A were negative.

A diagnosis of WDPM was made, based on histomorphology and IHC. A CT scan of the chest revealed no lesions in thoracic cavity. The patient was advised follow-up without chemotherapy. His postsurgical follow-up till 9 months was uneventful.

\section{Discussion}

WDPM is an uncommon variant of mesothelial neoplasm with uncertain malignant potential. It primarily occurs in the peritoneum of young women in their reproductive age group. Other sites such as pericardium, pleura, and tunica vaginalis have also been described. The long-term survival is good as pointed out by many studies and recurrences are also rare.

The differential diagnosis of WDPM includes atypical mesothelial hyperplasia, malignant mesothelioma, and adenocarcinoma with diffuse peritoneal involvement. The differentiation is really important due to vast differences in management and prognosis. Most primary peritoneal adenocarcinomas are papillary serous carcinomas and show the presence of cells with high-grade to bizarre nuclear features and numerous mitotic figures, both of which are absent in WDPM. Histochemical and IHC stains can help in the differential diagnosis with adenocarcinoma. In contrast to adenocarcinomas, WDPMs elaborate acid mucin rather than neutral mucin. The former is predominantly hyaluronic acid and is seen as alcian blue-positive and digested periodic acid-Schiff (DPAS)-negative material

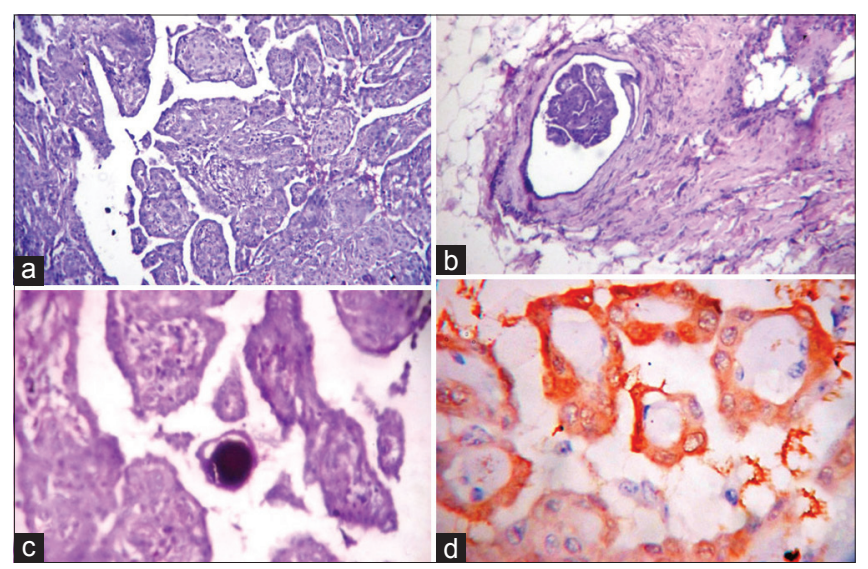

Figure 2: $(a, b, d)$ Photomicrograph showing histology of mesothelioma showing broad papillae with edematous fibrous cores and lined by uniform cuboidal cells with vascular invasion (b) and psammoma bodies (d), (H and E stain, $\times 10$ view). (c) The neoplastic cells are calretinin positive ( $\mathrm{IHC}$ stain, $\times 40$ view)

within cytoplasmic vacuoles ${ }^{[6]}$ The cell borders and stroma of WDPM may be DPAS positive. Immunoreactivity for both cytokeratin and vimentin, along with calretinin and WT-1 as well as negative staining for carcinoembryonic antigen and Ber-EP4, favors a diagnosis of mesothelioma over carcinoma.

Malignant mesothelioma can be differentiated from WDPM by their high-grade nuclear features, presence of necrosis, and mitoses with higher proliferation index. Comin et al. found that positivity for caldesmon and calretinin along with negativity for estrogen receptor and Ber-EP4 strongly favors malignant mesothelioma over serous carcinoma ${ }^{[7]}$ Our case was radiologically mistaken for an adnexal tumor but confirmed by histopathology and IHC. Fluid cytology can be misleading too as it can falsely give a diagnosis of reactive etiology, which happened in our case.

However, there is no single diagnostic IHC marker to separate WDPMs from adenocarcinomas and malignant mesotheliomas, and the results of a panel of antibodies should be interpreted in conjunction with proper clinical history, radiological evaluation, and histopathological examination by hematoxylin and eosin stain for a final diagnosis.

\section{Conclusion}

WDPM is a neoplasm with uncertain malignant potential and is frequently diagnosed incidentally. This case has been presented due to its rarity and the importance to differentiate it from its aggressive mimickers which have a much poorer prognosis.

\section{Declaration of patient consent}

The authors certify that they have obtained all appropriate patient consent forms. In the form the patient(s) has/ 
have given his/her/their consent for his/her/their images and other clinical information to be reported in the journal. The patients understand that their names and initials will not be published and due efforts will be made to conceal their identity, but anonymity cannot be guaranteed.

\section{Financial support and sponsorship \\ Nil.}

\section{Conflicts of interest}

There are no conflicts of interest.

\section{References}

1. Goepel JR. Benign papillary mesothelioma of peritoneum: A histological, histochemical and ultrastructural study of six cases. Histopathology 1981;5:21-30.

2. Daya D, McCaughey WT. Well-differentiated papillary mesothelioma of the peritoneum. A clinicopathologic study of 22 cases. Cancer 1990;65:292-6.

3. Goldblum J, Hart WR. Localized and diffuse mesotheliomas of the genital tract and peritoneum in women. A clinicopathologic study of nineteen true mesothelial neoplasms, other than adenomatoid tumors, multicystic mesotheliomas, and localized fibrous tumors. Am J Surg Pathol 1995;19:1124-37.

4. Butnor KJ, Sporn TA, Hammar SP, Roggli VL. Well-differentiated papillary mesothelioma. Am J Surg Pathol 2001;25:1304-9.

5. Diaz LK, Okonkwo A, Solans EP, Bedrossian C, Rao MS. Extensive myxoid change in well-differentiated papillary mesothelioma of the pelvic peritoneum. Ann Diagn Pathol 2002;6:164-7.

6. Hammar SP, Bockus DE, Remington FL, Rohrbach KA. Mucin-positive epithelial mesotheliomas: A histochemical, immunohistochemical, and ultrastructural comparison with mucin-producing pulmonary adenocarcinomas. Ultrastruct Pathol 1996;20:293-325.

7. Comin CE, Saieva C, Messerini L. H-caldesmon, calretinin, estrogen receptor, and ber-EP4: A useful combination of immunohistochemical markers for differentiating epithelioid peritoneal mesothelioma from serous papillary carcinoma of the ovary. Am J Surg Pathol 2007;31:1139-48. 